\title{
Incorporating Vibrational Excitation in a Hybrid Particle-Continuum Method
}

\author{
Timothy R. Deschenes* and Iain D. Boyd ${ }^{\dagger}$ \\ Department of Aerospace Engineering, University of Michigan, Ann Arbor, MI, 48109 \\ and \\ Thomas E. Schwartzentruber ${ }^{\ddagger}$ \\ Department of Aerospace Engineering and Mechanics, University of Minnesota, Minneapolis, MN, 55455
}

\begin{abstract}
A modular particle-continuum (MPC) method is extended to model vibrational excitation to simulate hypersonic steady-state flows that exhibit regions of collisional nonequilibrium in a mainly continuum flow field. This method loosely couples a DSMC code to an implicit Navier-Stokes solver. By limiting our study to steady-state flows, both time-step and cell size are decoupled between methods. Control of statistical scatter and information transfer between modules is described. Hypersonic flow over a 2-D cylinder is simulated with different physical models. Results from DSMC, CFD, and the MPC method are presented and compared. The agreement in vibrational temperature between DSMC and the MPC method decreases as the size of the continuum domain increases, which may be due to the difference in macroscopic relaxation rates computed in DSMC and CFD. Other flow variables and surface properties remain in excellent agreement between DSMC and the MPC method. The MPC simulation results are obtained using less than half the computational time compared to full DSMC while also decreasing the memory requirements.
\end{abstract}

\section{Nomenclature}

$C_{h} \quad$ Heat transfer coefficient

$d \quad$ Diameter

E Energy

$f \quad$ Probability density function

$g \quad$ Relative velocity

$k \quad$ Boltzmann constant $\left[1.38 \times 10^{-23} \frac{\mathrm{m}^{2} \mathrm{~kg}}{\mathrm{~s}^{2} \mathrm{~K}}\right]$

$m$ molecular mass $[\mathrm{kg}]$

$n \quad$ Particle number density $\left[\frac{\text { particles }}{m^{3}}\right]$

$N \quad$ Quantized Levels

$p \quad$ Pressure $[\mathrm{Pa}]$

$P_{i} \quad$ Probability of the $i$ th state

$Q \quad$ Flow quantity

$q \quad$ Heat flux $\left[\frac{W}{m}\right]$

$R \quad$ Universal gas constant

$T$ Temperature $[K]$

$\lambda \quad$ Mean free path $[m]$

$\mu \quad$ Coefficient of viscosity

$\nu \quad$ Mean collision rate

\footnotetext{
*Graduate Student, Student Member AIAA. Email: thytimo@umich.edu.

${ }^{\dagger}$ Professor, Associate Fellow AIAA. Email: iainboyd@umich.edu.

¥Assistant Professor, AIAA Member. Email: schwartz@aem.umn.edu.
} 


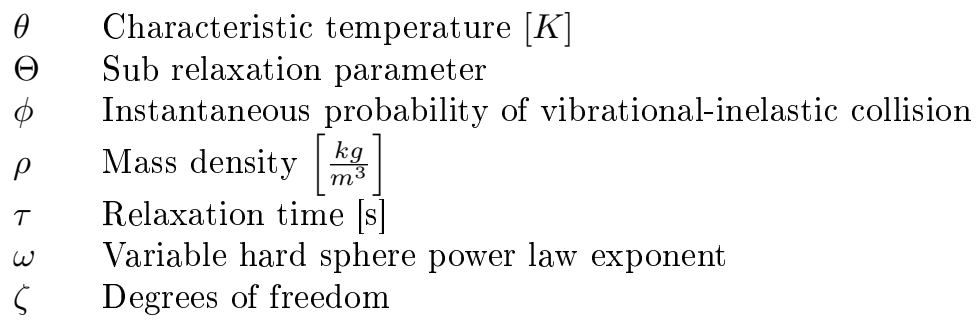

$\begin{array}{ll}\text { Subscripts } \\ \text { ABE Abe } \\ \text { BL } & \text { Borgnakke-Larsen } \\ \text { max } & \text { Maximum } \\ \text { GL } & \text { Gradient-length } \\ \text { ref } & \text { Reference state } \\ \text { rot } & \text { Rotational } \\ \text { tra } & \text { Translational } \\ v i b & \text { Vibrational } \\ w & \text { Wall } \\ \infty & \text { Free stream }\end{array}$

Subscripts

, Post collision

\section{Introduction}

With the increased interest in hypersonic vehicles, for scientific, defense, and commercial uses, there is a need for efficient and accurate computation of the flow field, aerodynamics, and heat transfer. Throughout the flight regime, hypersonic vehicles experience flow fields that can range from being completely rarefied to continuum. At very high altitudes, the flow is entirely rarefied, or in collisional nonequilibrium, while at low altitudes, the flow could be considered thoroughly continuum. Mature methods exist that are capable of computing rarefied or continuum flows. The direct simulation Monte Carlo (DSMC) method is often used to simulate highly rarefied flow conditions. ${ }^{1}$ Although accurate over the entire flow regime, the computational expense of performing DSMC on high density flow fields becomes prohibitively expensive due to restrictions on cell sizes and time-steps. In the continuum regime, the Navier-Stokes (NS) equations are applicable and can be solved accurately and efficiently using modern Computational Fluid Dynamics (CFD) techniques. In addition, models are available for both methods to account for excited vibrational modes and chemical reactions that can become important in flows that involve strong shocks. At intermediate altitudes, a flow can be characterized as mainly continuum with localized areas, such as the shock, the boundary layer, and the wake, that display collisional nonequilibrium effects. In the local rarefied regions, the Navier-Stokes equations are not accurate. While DSMC is accurate over the entire flow field, it is prohibitively expensive. Instead, a hybrid code can be used that solves the flow field with CFD in the continuum region and uses DSMC in the locally rarefied regions. Speedup can be achieved since restrictions imposed by DSMC, specifically the cell size must remain smaller than the mean free path and the time-step must remain less than the mean free time, are not required for CFD calculations. Thus, decoupling of both mesh densities and time-step are employed. This hybrid method can take advantage of the computational efficiency of CFD in regions where it is applicable, while still maintaining the physical accuracy of DSMC in the regions where the Navier-Stokes equations break down.

Previous research has been performed on "zonally decoupled" DSMC-CFD simulations. ${ }^{2-4}$ Here, a CFD simulation is performed up to a predetermined interface, then this information is used as the boundary condition for a DSMC simulation over the remainder of the domain. Small locally rarefied regions cannot be easily separated from mainly continuum flow since information transfer occurs in purely one direction and the CFD solution is completely decoupled from DSMC. Often the rarefied regions are very localized so various 
methods have been proposed that adaptively re-position the interface between DSMC and CFD throughout the simulation with various methods of coupling the domains. ${ }^{5-8}$ Wijesinghe and Hadjiconstantinou present a discussion of the major considerations involved as well as a summary of published work on coupled hybrid atomistic-continuum schemes. ${ }^{9}$ The present article extends the modular particle-continuum (MPC) method that was first developed for 1-D shock waves and extended to 2-D and axis-symmetric flows. ${ }^{10-12}$ In the previous work, the MPC method was able to limit DSMC to locally rarefied regions that occur within the shock, the boundary layer, and the wake of hypersonic blunt-body flows. By only simulating regions of collisional nonequilibrium with DSMC and decoupling time-steps and mesh densities between DSMC and CFD, the MPC method achieved speedup factors up to 3.27 for transitional flow. ${ }^{11,13}$ At higher densities, the locally rarefied regions become smaller and the MPC method achieved speedup factors greater than $10 .^{13}$

This article outlines the extension of the modular particle-continuum method by incorporating vibrational excitation, which occurs in high enthalpy flows. Control of the statistical scatter and transfer of information between the DSMC and CFD modules is outlined and extended with the addition of vibrational energy. Hybrid simulations of the steady-state flow about a hypersonic blunt-body are performed with the MPC method with vibrational relaxation and comparison with corresponding full DSMC and full CFD solutions is performed. In addition, comparison of flow field features between simulations with rotational nonequilibrium and forced rotational equilibrium in DSMC regions are compared and the effect on the solution of other flow variables with the MPC method are discussed. Conclusions are formulated and areas of future work are discussed.

\section{Modular Particle-Continuum Method}

The MPC method uses existing, state of the art, DSMC and CFD modules with the addition of procedures to determine the interface location and transfer of information between the two methods. This section briefly summarizes the code structure while covering the details of the added vibrational energy information transfer. Further details of the MPC algorithm can be found in Reference 12.

\section{II.A. DSMC and CFD Modules}

In particle regions, the MPC algorithm uses MONACO, ${ }^{14}$ a general, cell-based implementation of the DSMC method capable of simulating rotational and vibrational nonequilibrium and multi-species flow with finite rate chemistry. The variable hard sphere model is employed which results in the following macroscopic viscosity-temperature dependence:

$$
\begin{gathered}
\mu=\mu_{\text {ref }}\left(\frac{T}{T_{r e f}}\right)^{\omega} \\
\mu_{\text {ref }}=\frac{15 \sqrt{\pi m k T_{r e f}}}{2 \pi d_{r e f}^{2}(5-2 \omega)(7-2 \omega)}
\end{gathered}
$$

All results presented in this article are for diatomic oxygen with a reference diameter of $d_{\text {ref }}=4.07 \times$ $10^{-10} \mathrm{~m}$ at $T_{\text {ref }}=273 \mathrm{~K}$. The power law exponent used is $\omega=0.75$ while $\mathrm{m}$ is the mass of an $O_{2}$ molecule and $k$ is the Boltzmann constant. MONACO employs the variable rotational energy exchange probability of $\mathrm{Boyd}^{15}$ where the reference temperature for energy exchange is $T_{\text {ref,rot }}=91.5 \mathrm{~K}$ and the maximum collisional number is 18.1 .

In continuum regions, the MPC method uses the LeMANS code ${ }^{16}$ developed at the University of Michigan. LeMANS is capable of simulating 2-D, axis symmetric, or 3-D steady state continuum hypersonic flows with vibrational nonequilibrium. LeMANS uses a finite-volume formulation to solve the Navier-Stokes equations. The inviscid fluxes are calculated using a modified form of the Steger-Warming Flux Vector Splitting. The modified form is less dissipative which makes it adequate to solve the boundary layer, while a pressure switch is used to switch back to the original form near shock waves. The viscous terms are calculated using the values of properties at the cell centers and at the nodes. Time integration is performed using a point-implicit method. Reference 16 contains more specific details of the numerical procedures used in the CFD module. Currently, translation-rotation nonequilibrium is ignored in the CFD module. Viscosity in CFD regions is set to match the viscosity produced by the variable hard sphere model used in DSMC, shown in Eq. 1.

Translation-vibration relaxation is incorporated using a relaxation time in the form of Eq. 3, where $\tau_{v i b}$ is the continuum relaxation time in seconds and $p$ is the pressure in Pascals. 


$$
\tau_{v i b}=\frac{1}{p} \exp \left[A T_{t r a}^{-\frac{1}{3}}+B\right]
$$

The constants used are those suggested for $O_{2}$ by Millikan and White and are $A=129$ and $B=-10.76{ }^{17}$ Simulations with and without Park's high temperature correction were performed, ${ }^{18}$ but due to the relatively low flow-field translational temperature, it had no noticeable affect on any flow variables. The direct application of the relaxation time is used in the CFD module, while it must be converted to an instantaneous probability based on relative velocity between two colliding particles for the DSMC module.

The average probability of a vibrationally-inelastic collision, $\bar{P}$, can be expressed in terms of the vibrational relaxation time, $\tau_{v i b}$, and the mean collision rate, $\nu$ as shown in Eq. 4 . The average probability can be related to the instantaneous probability, $\phi(g)$, as seen in Eq. 5 where $g$ is the relative velocity between two particles and $f(g)$ is the relative velocity distribution function. A modified Landau-Teller form for the instantaneous probability as seen in Eq. 6 and a Boltzmann velocity distribution is assumed. The constants, $Z_{o}, \alpha$, and $g^{*}$ are evaluated in terms of the variable hard sphere model and Millikan and White coefficients so that Eq. 5 is satisfied. This integral cannot be solved exactly so the method of steepest decent is used. ${ }^{19}$

$$
\begin{gathered}
\bar{P}=\frac{1}{\tau_{v i b} \nu} \\
\bar{P}=\int_{0}^{\infty} \phi(g) f(g) d g \\
\phi(g)=\frac{1}{Z_{o}} g^{\alpha} \exp \left(\frac{-g^{*}}{g}\right)
\end{gathered}
$$

In addition, there are differences in how the relaxation time is defined in particle and continuum simulations. Lumpkin, Haas, and Boyd studied these differences for rotational relaxation. Their result can also be applied to vibrational relaxation and the relation between particle and continuum relaxation times as Eq. 7 , where $\zeta_{v i b}$ is the number of degrees of freedom of the vibrational energy mode and $\omega$ is the power law exponent for the VHS model. Therefore, the instantaneous probability for vibrationally-inelastic collision is multiplied by a factor of $1+\frac{\zeta_{v i b}}{4-2 \omega}$, for consistency with the CFD model. A value of $\zeta_{v i b}$ is calculated based on a maximum temperature of $8,000 \mathrm{~K}$.

$$
\tau_{v i b}^{D S M C}=\frac{\tau_{v i b}^{C F D}}{1+\frac{\zeta_{v i b}}{4-2 \omega}}
$$

MONACO is also capable of taking into account Park's high temperature correction term, but that is not included in these simulations since translational temperatures remain relatively low throughout the entire flow field. The maximum translational temperature remains below 7,500 $K$ and Park suggests that Millikan and White data should only be modified for translational temperatures greater than $8,000 K{ }^{18}$

Despite all attempts to maintain similar relaxation times between DSMC and CFD under collisional equilibrium conditions, the average probabilities simulated in each method still are not exactly the same. Table 1 shows a comparison between the probabilities of vibrationally-inelastic collisions in a heat bath sampled using MONACO simulations to exact calculations. Although remaining within $20 \%$ across the entire flow field of interest, these differences still influence agreement in vibrational temperature contours between DSMC and CFD in continuum simulations. It is thought that these differences are due to the inexact integration of Eq. $5 .^{20-22}$

Table 1. Comparison of average probabilities of vibrationally-inelastic collisions between DSMC and theory for $\mathrm{O}_{2}-\mathrm{O}_{2}$ collisions. ${ }^{20}$

\begin{tabular}{|c|c|}
\hline$T_{\text {tra }}[K]$ & $\frac{\bar{P}_{M O N A C O}}{\bar{P}_{\text {THEORY }}}$ \\
\hline \hline 5,000 & 1.16 \\
\hline 10,000 & 0.82 \\
\hline 15,000 & 0.80 \\
\hline 20,000 & 0.83 \\
\hline 25,000 & 0.85 \\
\hline
\end{tabular}


Finally, post-collision vibrational energy is sampled from a Borgnakke-Larsen energy distribution with a correction proposed by $\mathrm{Abe}^{23}$ that maintains the principle of detailed balance. Equation 8 shows the relation between the energy distribution proposed by Abe and the energy distribution function proposed by Larsen and Borgnakke where $f_{A B E}$ is the distribution used to sample post-collisional vibrational energy, $f_{B L}$ is the discrete Borgnakke-Larsen energy distribution, ${ }^{24} \phi_{\max }$ is the maximum probability of vibrationally-inelastic collision based on post-collision translational energy, and $g^{\prime}$ is the post-collision relative velocity.

$$
f_{A B E}=\frac{\phi\left(g^{\prime}\right)}{\phi_{\max }} \times f_{B L}
$$

\section{II.B. Interface Location and Continuum Breakdown}

The accuracy and efficiency of a hybrid DSMC-CFD method strongly depend on proper placement of the interface location. The interface location must be located within collisional equilibrium regions to ensure that the NS solver maintains acceptable physical accuracy over its entire domain, while the efficiency of the MPC method depends on the interfaces remaining near the edge of the collisional equilibrium domain. Often, a continuum breakdown parameter is used to determine the particle and continuum regimes of the flow field. The MPC method employs the gradient-length Knudsen number first proposed by Boyd et al. ${ }^{25,26}$ as seen in Eq. 9 where $Q$ is a flow quantity of interest such as density $\rho$, pressure $p$, temperature $T$, or velocity magnitude $|V|$, and $\lambda$ is the local mean free path.

$$
K n_{G L}=\frac{\lambda}{Q}|\nabla Q|
$$

Previous research has shown for hypersonic reentry problems ${ }^{25,26}$ and 1-D normal shock waves ${ }^{10}$ that regions where the maximum gradient-length Knudsen number remain less than 0.05 display less than a $5 \%$ discrepancy of flow quantities between DSMC and CFD. Therefore, these regions may be simulated with CFD while introducing little physical inaccuracy.

In addition, since the CFD module currently does not allow rotational nonequilibrium, an added breakdown parameter that measures the degree of rotational nonequilibrium in the flow is used. Equation 10 is applied without absolute values so that only areas where $T_{\text {tra }}$ exceeds $T_{\text {rot }}$ by more than $1 \%$ are simulated using DSMC while areas where $T_{\text {rot }}$ exceeds $T_{\text {tra }}$ are allowed to be simulated using CFD.

$$
\begin{gathered}
K n_{R O T-N E Q}=5 \times \frac{T_{\text {tra }}-T_{\text {rot }}}{T_{\text {tra }}} \\
K n=\max \left(K n_{G L-\rho}, K n_{G L-p}, K n_{G L-T_{T R A}}, K n_{G L-|V|}, K n_{R O T-N E Q}\right)
\end{gathered}
$$

Equation 11 shows the final breakdown parameter used in the MPC method which is a combination of the two parameters described above. Figure 1 shows the initial and final interface locations for a Mach 12 case described in Section III.

\section{II.C. Information Transfer}

The modular implementation of the MPC method allows both the DSMC and CFD modules to maintain their own mesh and data structure. State-based coupling is performed which assigns boundary conditions to the CFD domain from corresponding DSMC cells and vice versa. Figure 2 shows a schematic of how data is transferred between the two modules at an interface location. First, the interface locations are determined using the breakdown parameter evaluated for a full CFD solution. Next, a DSMC buffer region is created and cells that are used for boundary conditions are added to the edges of the particle and continuum domains.

An unsteady period occurs where the flow field changes from the initial CFD solution to a hybrid particlecontinuum result. During this time, the interfaces are allowed to move. In this unsteady phase, macroscopic information is tracked in DSMC cells using a sub-relaxation average proposed by Sun and Boyd ${ }^{27}$ and is shown in Eq. 12 where $\bar{Q}_{j}$ is the average at the current iteration, $\bar{Q}_{j-1}$ is the average at the previous iteration, $Q_{j}$ is the value at the current iteration, and $\Theta$ is the weighting used. A $\Theta$ value of 0.001 is used throughout all the MPC simulations presented in this article.

$$
\bar{Q}_{j}=(1-\Theta) \bar{Q}_{j-1}+\Theta Q_{j}
$$




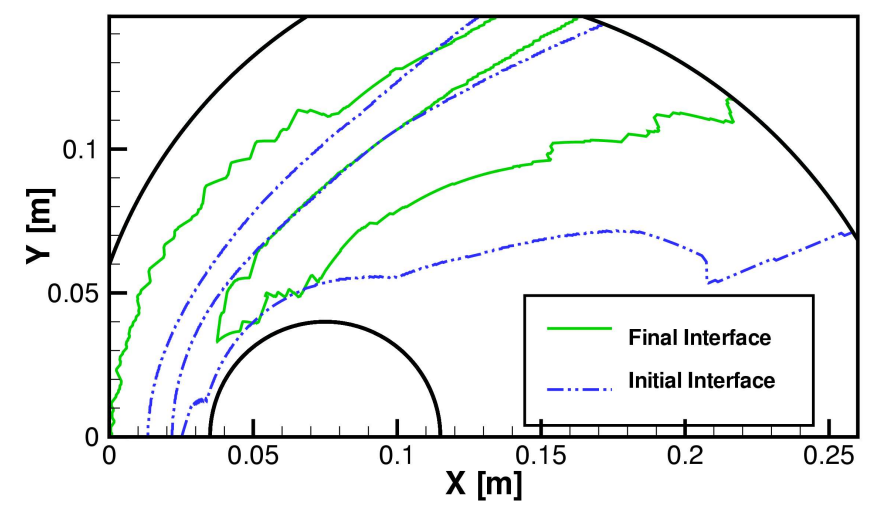

Figure 1. DSMC-CFD interface locations.

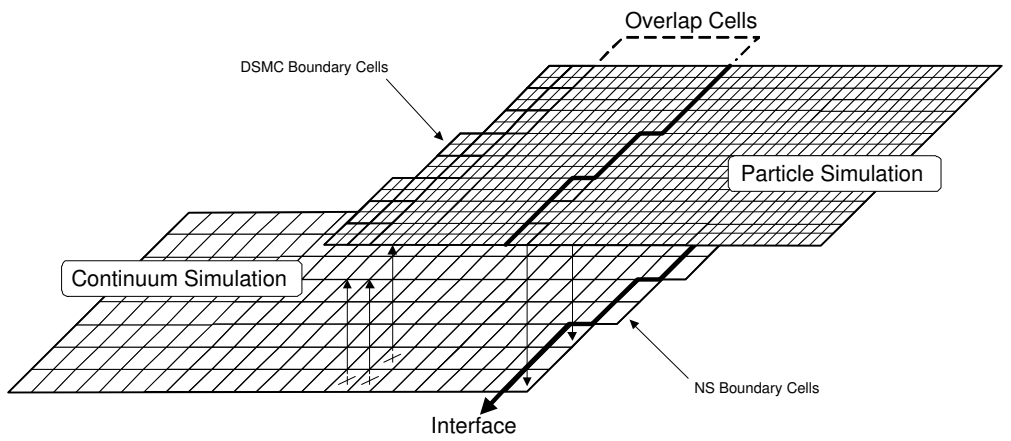

Figure 2. State-based coupling.

Although the sub relaxation technique reduces the scatter of macroscopic flow variables, it should only be used if the flow variables of interest are resolved to acceptable levels of statistical scatter in less than $\frac{1}{\Theta}$ iterations. For most flow variables, this can often be done in very few iterations. For vibrational temperature, a quantized energy distribution is used in the DSMC module and the number of iterations required to resolve low vibrational temperatures becomes enormous. It is found that the number of iterations required to accurately resolve vibrational temperature varies inversely with the ratio $\frac{T_{v i b}}{\theta_{v i b}}$ where $\theta_{v i b}$ is the characteristic temperature for vibration and $T_{v i b}$ is the vibrational temperature being sampled. Figure 3 compares the temperature at a point in a shock layer (left), where the vibrational temperature is about $3,000 K$, and at a point upstream of the shock (right), where the vibrational temperature is $217.45 \mathrm{~K}$, as a function of the number of iterations. When the vibrational temperature is on the same order of magnitude of $\theta_{v i b}$, where $\theta_{v i b}=2239 \mathrm{~K}$ for $\mathrm{O}_{2}$, acceptable resolution of the vibrational temperature occurs in about the same number of iterations required by both translational and rotational temperature. However, at low vibrational temperature, an excessively large number of time-steps are required to resolve the vibrational temperature. This is because the probability of a particle being vibrational excited at low temperatures is extremely low. Even after 30,000 iterations with more than 30 particles per iteration, the vibrational temperature can not be accurately resolved and only 4 particles with nonzero energy have passed through the cell. As a result of the infrequency of vibrationally excited particles at low temperatures, the sub-relaxation average would 
require an enormous number of samples to converge to the correct value of $217.45 \mathrm{~K}$. This is prohibitive for efficient simulation using the MPC method.

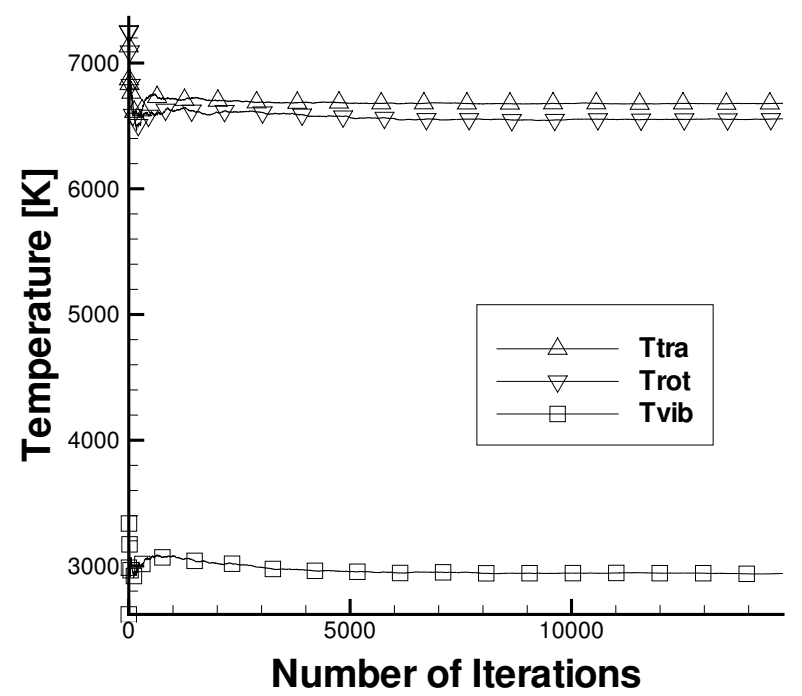

(a) Postshock Location.

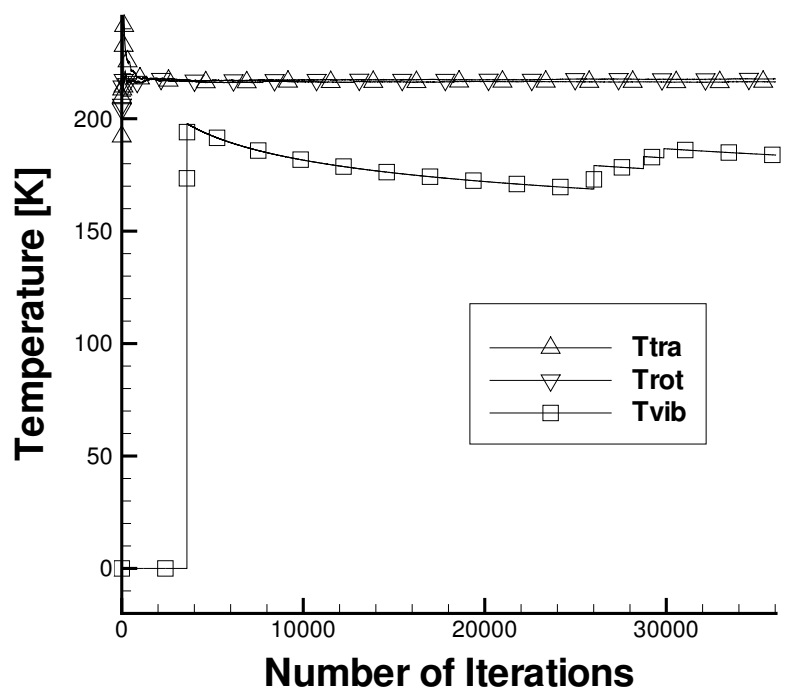

(b) Preshock Location.

Figure 3. Comparison of the number of iterations needed to resolve the temperatures in a post-shock (left) and pre-shock (right) locations.

To address the problem illustrated in Fig. 3 of efficient resolution of low vibrational temperatures, the average vibrational energy is assigned to all particles in the boundary cells. Equation 13 shows the final calculation of the vibrational energy where $N_{\max }$ is the level that the discrete Boltzmann distribution is truncated in the DSMC simulation, $R$ is the universal gas constant, $\theta_{v}$ is the characteristic vibrational temperature, and $P_{i}$ is the probability of a particle occupying the $i$ th level of vibrational energy. Assuming vibrational energy is modeled as a harmonic oscillator, the probability of obtaining a level is calculated using Eq. 14. The maximum level, $N_{\max }$, is calculated using Eq. 15 where $P_{i, \min }$ is the minimum probability sampled in the DSMC simulation. Currently, MONACO samples probabilities as low as $10^{-8}$, so this is used as $P_{i, \min }$ for all simulations presented.

$$
\begin{gathered}
E_{v i b}=\sum_{i=0}^{N_{\max }} P_{i} i \theta_{v i b} R \\
P_{i}=\exp \left[\frac{-i \theta_{v i b}}{T_{v i b}}\right]\left(1-\exp \left[\frac{-\theta_{v i b}}{T_{v i b}}\right]\right) \\
N_{\text {max }}=i n t\left(-\ln \left(P_{i, \text { min }}\right) \frac{T_{v i b}}{\theta_{v i b}}\right)
\end{gathered}
$$

DSMC collision process described above remain intact, so particles are assigned to quantized levels after they undergo a vibrationally-inelastic collision. Using this method, vibrational temperature information passed from the DSMC module to the CFD module uses the same relaxation process used by all other variables as defined in Eq. 12. This new method is tested in heat bath and 2-D flow simulations using DSMC. All macroscopic values remain in good agreement with the original discrete population method. As will be seen in Section IV, results derived by application of this method in the interior of the flow in a hybrid simulation agree well with full DSMC simulations as well. 


\section{Flow Conditions}

Hypersonic flow about a cylinder at Mach 12 is simulated. The free-stream gas simulated is $\mathrm{O}_{2}$ with number density $n_{\infty}=1.41 \times 10^{21}$ particles $/ \mathrm{m}^{3}$ and $T_{\text {tra }, \infty}=T_{\text {rot }, \infty}=T_{v i b, \infty}=217.45 \mathrm{~K}$. This corresponds to a free stream density of $\rho_{\infty}=7.48 \times 10^{-5} \mathrm{~kg} / \mathrm{m}^{3}$ and pressure of $p_{\infty}=4.22 \mathrm{~Pa}$. The diameter of the cylinder simulated is $d=8 \mathrm{~cm}$ which results in a global Knudsen number of $K n_{\infty}=0.01$. The cylinder wall temperature is set at $T_{w}=1000 \mathrm{~K}$ with full diffuse reflection in DSMC simulations and a no-slip, isothermal condition is applied in CFD simulations. The vibrational characteristic temperature used in all simulations is $\theta_{v i b}=2239 \mathrm{~K}$. These flow conditions are very similar to those used previously in Reference 13 with the exception of using $\mathrm{O}_{2}$ instead of $N_{2}$. This change is made to increase the ratio of the maximum flow temperature to the vibrational characteristic temperature which can be used as an indication of the amount of vibrational excitation expected in the flow. A constant DSMC time-step of $2 \times 10^{-8} s$ is used for all simulations.

Results with different physical models and mesh structures are compared. Case M12 corresponds to the baseline case where both the DSMC and CFD modules are unmodified. In addition, both DSMC and CFD use the same structured mesh that is refined to the mean free path. Case M12 $\mathbf{E}$ corresponds to a flow simulation using the same mesh used in case M12 but sets the rotational collision number to unity in the DSMC module. This forces the rotational temperature to be closer to equilibrium with the translational temperature in flow areas that are near collisional equilibrium. Finally, a third case $\mathbf{M} 1 \mathbf{2}_{\mathbf{R}}$ uses the same physical models as case M12 but the CFD module uses a much coarser mesh and the mesh refinement technique explained in Ref. 13 is employed.

\section{Numerical Results of Flow Simulations}

Ultimately, the purpose of the MPC method is to reproduce full DSMC results at a lower computational expense. This section will compare flow field results between cases M12 and M12 ${ }_{E}$. Flow field properties for case $\mathbf{M} 12_{\mathbf{R}}$ are in excellent agreement with case $\mathbf{M 1 2}$, so therefore are not be shown. In addition, surface properties and code efficiency are compared to the corresponding full DSMC simulations for all three cases.

\section{IV.A. Flow Field Properties}

Figure 4(a) compares the translational temperature contours calculated using full DSMC, full CFD, and the MPC method for the M12 case. The DSMC shock wave is significantly thicker and begins further upstream compared with the CFD shock profile. In addition, the DSMC solution predicts a temperature overshoot within the shock wave. The wake region also shows that the temperature predicted by DSMC is up to $20 \%$ higher than the temperature predicted by CFD. Figure 4(b) compares the vibrational temperature contours calculated using full DSMC, full CFD, and the MPC method for the M12 case. DSMC predicts a thicker vibrational relaxation area in the fore-body, which directly corresponds to the thicker translational temperature shock seen in Figure 4(a), in the forebody compared to full CFD and also a higher vibrational temperature in the expansion and wake area compared to DSMC.

Figure 4(a) also shows that the MPC method is able to reproduce nonequilibrium effects that CFD is unable to simulate alone, including the temperature overshoot in the shock, the thicker shock structure, and the higher translational temperature within the near-wake region. The maximum difference for translational temperature when comparing the MPC results to full DSMC is less than $3 \%$.

In addition, Figure 4(b) shows that the MPC method successfully improves from the initial NS solution to a result that compares well with full DSMC. The MPC solution is able to reproduce the higher vibrational temperature in the expansion and wake that CFD is unable to predict and also the larger relaxation zone located in the forebody. The largest difference between MPC and DSMC flow field calculations for vibrational temperature is seen to be located in the far wake region with a percentage difference of about $10 \%$.

Notice that the areas that show greatest differences in vibrational temperature are located in the wake that also corresponds to the area with the greatest differences in translational temperature which are due to rotational nonequilibrium effects. Since case $\mathbf{M 1 2}$ E pushes the flow towards rotational-translational equilibrium, better agreement in translational temperatures are obtained between DSMC and the MPC method. This is because DSMC and CFD are in better agreement for translational temperature in areas which are considered continuum. Figure 5 compares vibrational temperature contour lines between the MPC method and DSMC for cases M12 (top) and M12 (bottom). Surprisingly, the agreement for vibrational 


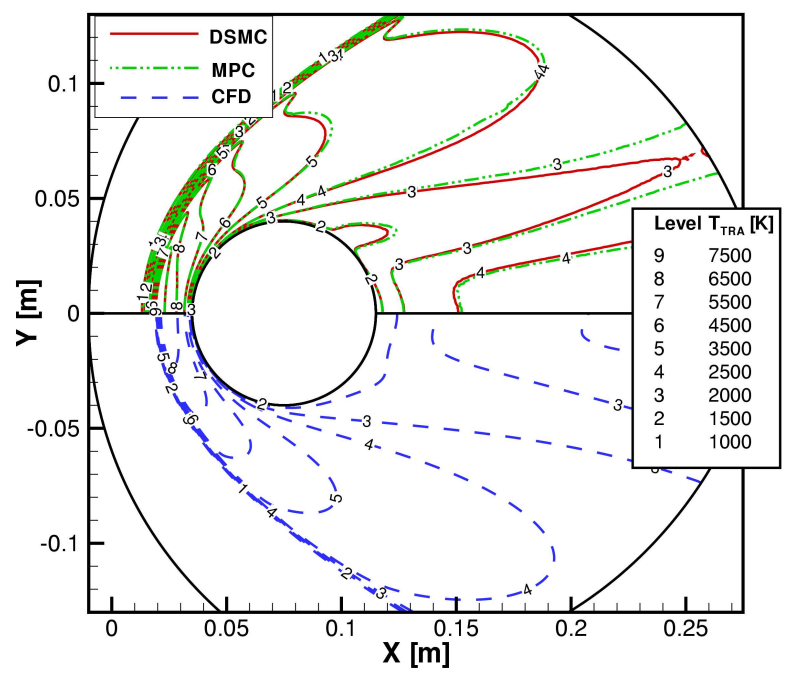

(a) Translational temperature.

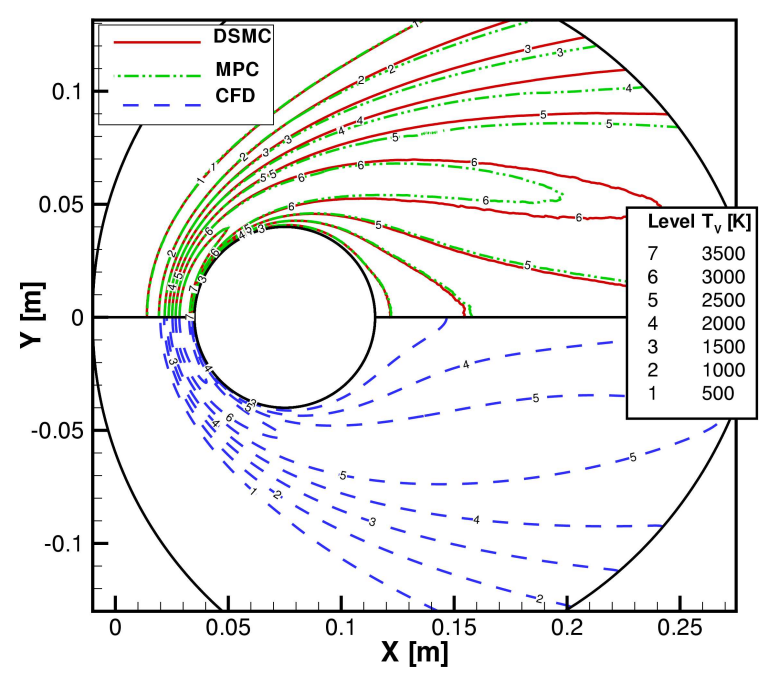

(b) Vibrational temperature.

Figure 4. Temperature contour comparison between DSMC, CFD, and the MPC for the M12 simulation.

temperature between the MPC method and full DSMC actually worsens even though all other flow variables are in better agreement for the $\mathbf{M 1 2}$ E case compared to the M12 case. In order to better quantify these differences, extraction line cuts with the angle measured from the extraction line are made that compare the flow field temperatures predicted with CFD, DSMC, and the MPC method for the M12 and M12 $\mathrm{E}$ cases.

In the fore-body, the flow is dominated by nonequilibrium effects and almost the entire flow is simulated by the MPC using the DSMC module which results in excellent agreement. Figure 6 (a) and (b) show the temperature profiles along the stagnation line and $45^{\circ}$ extraction line measured from the stagnation streamline for the M12 case, respectively. Since the entire area of interest is simulated using DSMC in the MPC method, the MPC results are in excellent agreement with full DSMC results. Similar agreement is found for the $\mathrm{M}_{\mathrm{E}}$ case.

The largest differences start to appear where the flow begins to expand around the cylinder and the local translational and rotational temperatures drop below the vibrational temperature. Figure 7 compares temperatures between CFD, MPC, and DSMC along the $90^{\circ}$ extraction line. Along the $90^{\circ}$ extraction line, one can see that there is a continuum domain located between two particle regions that contain the boundary layer and bow shock. The size of the region where the CFD module is applied increases in the $\mathrm{M12}_{\mathrm{E}}$ case due to the fact that rotation-translation equilibrium is attained over a much shorter distance. Despite improved agreement in both translational and rotational temperature for the $\mathbf{M} \mathbf{1 2} \mathbf{E}$ case (right), the agreement between vibrational temperatures actually worsens between MPC and DSMC results. Similar characteristics are seen along extraction lines at $135^{\circ}$ and $165^{\circ}$ in Figures 8 and 9, respectively, despite noticeable improvement in translational and rotational temperatures. Notice that along the $135^{\circ}$ extraction line, a discontinuity exists in vibrational temperature at the DSMC-CFD interface. This only appears along the DSMC-CFD interface in MPC simulations where there is also a discontinuity in translational temperature. Also, the size of the vibrational discontinuity is proportional to the size of the translational temperature discontinuity. It is thought that if rotational nonequilibrium were accounted for in CFD simulations, the discontinuity in translational temperature would be eliminated which would also eliminate the vibrational temperature discontinuity. Also even though the $165^{\circ}$ extraction line is located entirely within a DSMC region, differences in vibrational temperature still exist. Despite differences away from the body, DSMC and the MPC method are in excellent agreement near the surface of the body.

A possible cause of the increased discrepancy in vibrational temperature between DSMC and MPC results could be due to the inherent differences in macroscopic vibration-translation relaxation rates simulated by 


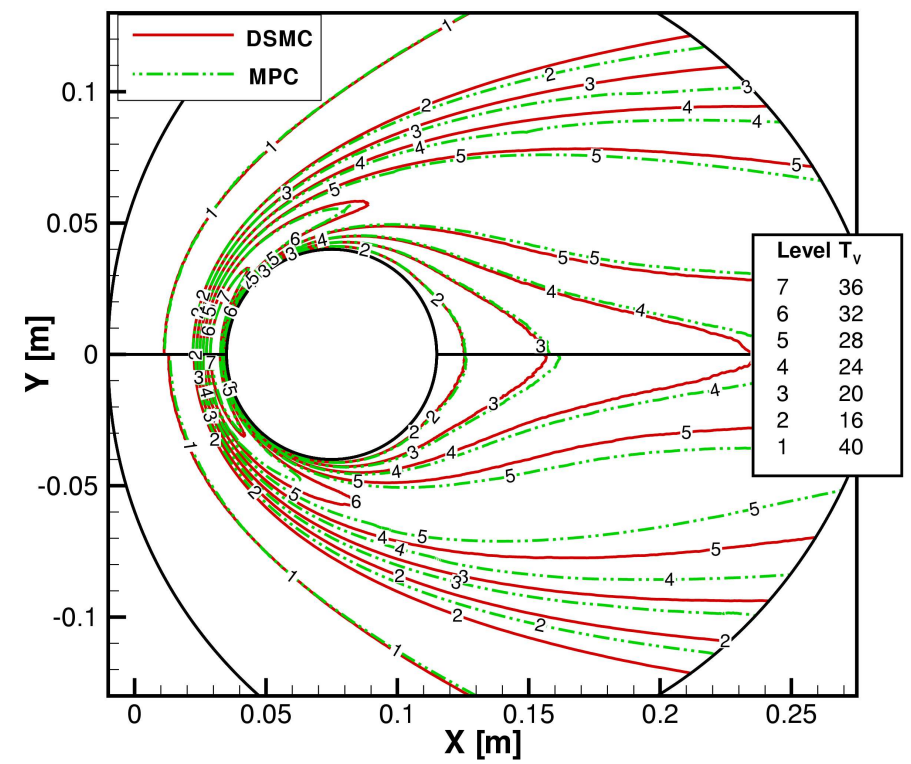

Figure 5. Comparison of vibrational temperature contour lines for case M12 (top) and M12E (bottom).

CFD and DSMC. As noted before, although the method used in DSMC was formulated to match continuum relaxation rates in collisional equilibrium areas, exact replication of rates does not occur. ${ }^{19,20,28}$ Figure 10 show streamlines that pass through areas that exhibit the highest discrepancy in vibrational temperature between DSMC and the MPC method. Notice that the areas that display largest differences in vibrational temperature also pass through the continuum region. To the rear of the shock, the flow contained between streamlines in Figure 10 can be considered advection-dominated so that most fluid elements (in CFD regions) or particles (in DSMC regions) will nearly follow the streamlines. This causes some areas of DSMC flow to be strongly influenced by information from the continuum regions. In addition, since the forced rotationaltranslational equilibrium extends the continuum region further into the shock layer, more fluid elements pass through the continuum region which results in larger discrepancies in the wake region. Therefore, it is thought that the differences in vibrational relaxation rates are the main cause of the discrepancies in vibrational temperatures between DSMC and the MPC method.

\section{IV.B. Surface Properties}

Despite the differences in the temperature flow fields, surface properties are in excellent agreement. Figure 11(a) compares the coefficient of heating, $C_{h}$, for the M12 and M12 $\mathbf{R}$ cases from the MPC, full CFD, and full DSMC, where $C_{h}$ is defined in Eq. 16 and $q$ is the heat flux to the surface, $\rho_{\infty}$ is the free stream density, and $V_{\infty}$ is the free stream velocity. Figure 11(b) compares the coefficient of heating for the $\mathbf{M 1 2}$ ease from the MPC, full CFD, and full DSMC.

$$
C_{h}=\frac{q}{\frac{1}{2} \rho_{\infty} V_{\infty}^{3}}
$$

Notice that despite the differences in the vibrational temperature flow field away from the body, all MPC heat flux results are in excellent agreement with full DSMC simulations. The MPC method can accurately predict heating rates to within a maximum difference of less than $2 \%$ compared to full DSMC with a significant reduction in computational expense.

\section{IV.C. Computational Performance}

The MPC method reproduces full DSMC results while using a fraction of the particles required by limiting the DSMC method to regions that are in collisional nonequilibrium. Continuum regions are simulated using 


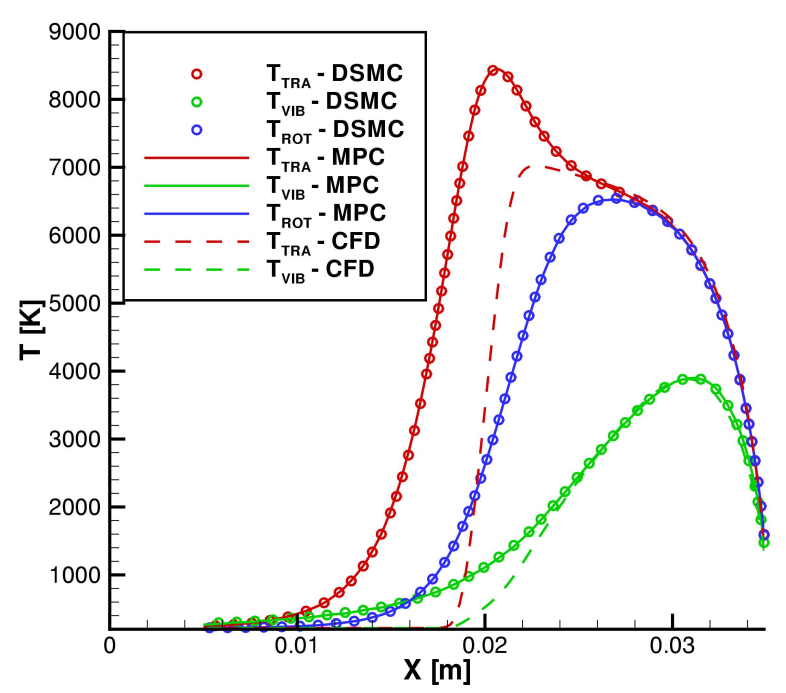

(a) Stagnation line.

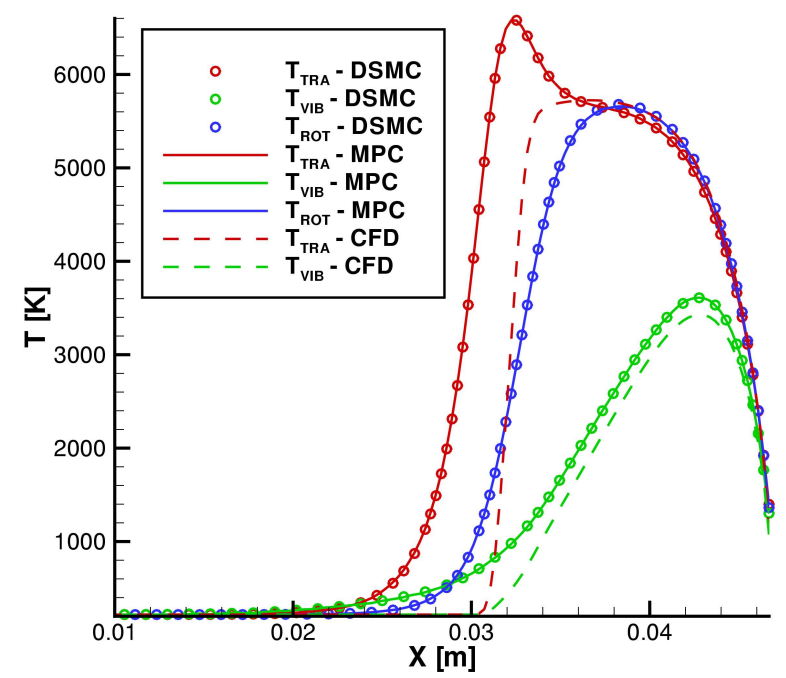

(b) $45^{\circ}$ extraction line.

Figure 6. Comparison of temperature profiles with rotational nonequilibrium in DSMC.

an implicit Navier-Stokes solver so time-steps restrictions that must be applied to DSMC simulations are not required. This allows continuum time-steps to be 50 times larger compared to the time-steps required by DSMC for the corresponding flow. In addition, for case $\mathbf{M} 12_{\mathbf{R}}$, the use of mesh refinement leads to a decoupling of mesh densities as well. For case $\mathbf{M} 12_{\mathbf{R}}$, continuum cells are up to 14 times larger in area than corresponding DSMC cells. The decreases in both computational time required and memory usage are outlined in Table 2. The actual speedup is the ratio of the computational time required by DSMC simulation compared to the corresponding simulation using the MPC method. To make a fair comparison, the number of sample time-steps after reaching steady-state are the same using DSMC and the MPC method. Ideal speedup is defined as the ratio of particles used in a full DSMC simulation compared to the corresponding MPC simulation. Since the computational cost of DSMC simulations nearly scales linearly with the number of particles in the simulation, if the overhead and continuum regions are completely free in the MPC method, this is the highest speedup factor achievable for a flow. Notice, that for all cases the actual speedup factor exceeds the ideal speedup factor. This is because the time required to reach steady state is greatly reduced in the MPC method due to using an initial continuum solution that can be calculated in much less computational time than the corresponding full DSMC simulation. For comparison, the sampling speedup factors are listed as well. These are calculated as the ratio of the time required to sample the same number of time-steps in a full DSMC simulation to that required for the MPC method. As noted before, these are always below the ideal speedup. Finally, memory usage is calculated as the ratio between the maximum memory required for an MPC simulation compared to the corresponding full DSMC simulation. Note that with the use of mesh refinement, a large decrease in memory usage is attainable. In addition, the $\mathbf{M} 1 \mathbf{2}_{\mathbf{E}}$ case achieved a higher speedup factor compared to the M12 case. This is because the size of the particle regions is reduced due to eliminating the need to apply the rotational nonequilibrium breakdown parameter, $K n_{R O T-N E Q}$, in areas that are near collisional equilibrium. Since current CFD methods can account for translational-rotational nonequilibrium, addition of this capability into the continuum module could lead to larger speedup factors.

\section{Conclusions}

The implementation of vibrational relaxation within a modular particle-continuum (MPC) method was described and tested on a hypersonic, blunt-body flow that is in transitional collisional nonequilibrium. An MPC method is outlined that uses existing DSMC and CFD codes with virtually no modification within a 


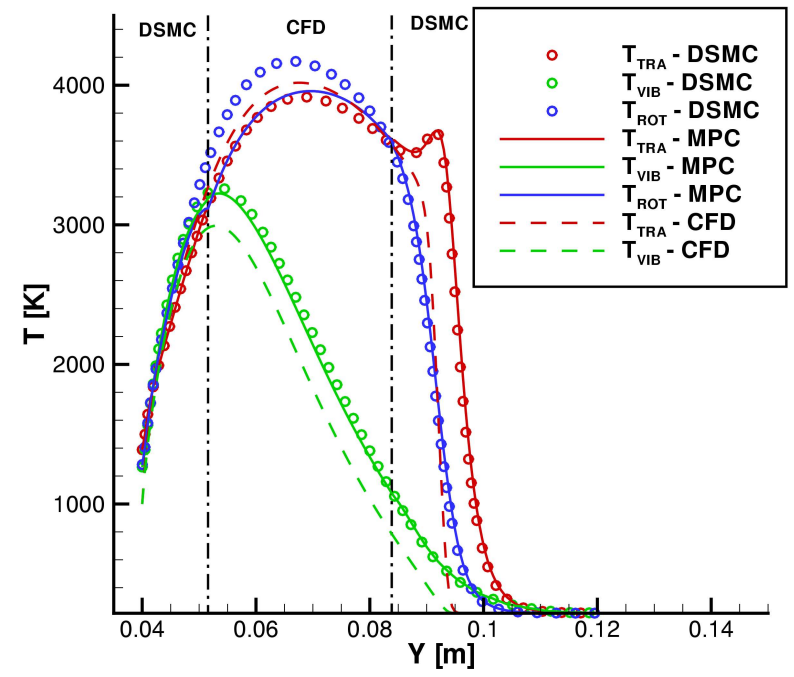

(a) M12 case.

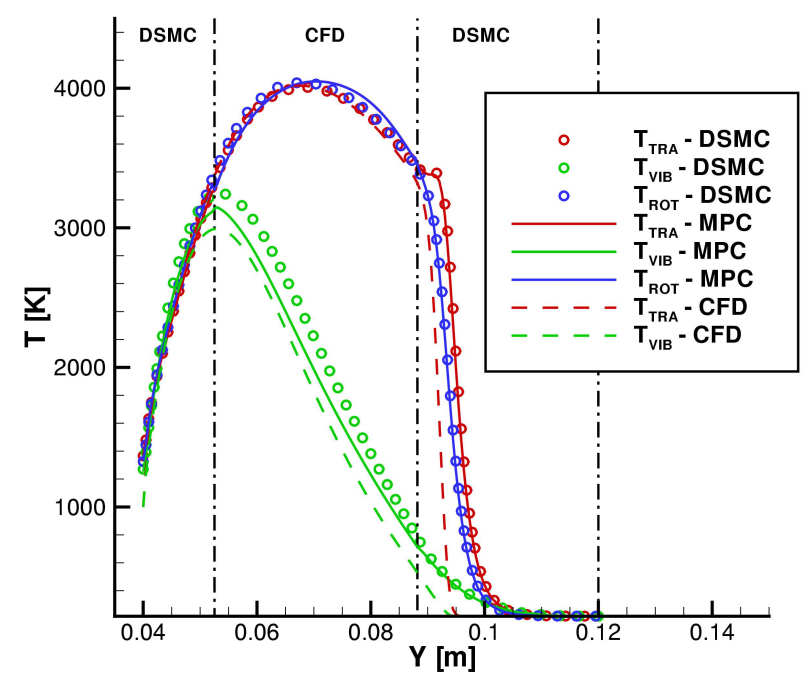

(b) $\mathrm{M} 12_{E}$ case.

Figure 7. Comparison of temperature profiles along the $90^{\circ}$ extraction line.

Table 2. Computational performance and memory requirements for the MPC method.

\begin{tabular}{|c|c|c|c|c|}
\hline Case & Actual Speedup & Ideal Speedup & Sample Speedup & Memory Usage \\
\hline \hline M12 & 2.29 & 1.75 & 1.68 & $98 \%$ \\
\hline M12 $_{\mathbf{E}}$ & 2.34 & 1.92 & 1.72 & $76 \%$ \\
\hline M12 $_{\mathbf{R}}$ & 2.59 & 2.29 & 1.90 & $59 \%$ \\
\hline
\end{tabular}

hybrid code. This implementation allows updates of both the DSMC or CFD source codes which reduces the development time of a hybrid code as newer models are added and tested to both codes. Information transfer techniques of flow variables between DSMC and CFD modules were extended to account for vibrational energy. A method for controlling the statistical scatter of vibrational temperature was described and tested. This enables the MPC method to handle information transfer including vibrational energy in an accurate and efficient manner. Comparison of flows with and without translation-rotational nonequilibrium activated in DSMC regions were performed. Despite differences of about $10 \%$ for the vibrational temperature in the wake region, surface properties calculated using the MPC method were in excellent agreement with full DSMC calculations. With the reduction in particles and the decoupling of both time-step and mesh densities, the MPC method is able to resolve nonequilibrium flow features using the computationally expensive DSMC method, while using CFD in continuum regions. The reduction in computational time is directly proportional to the reduction in number of particles and the MPC method significantly reduces the number of particles by decreasing the size of the DSMC domain to only areas in collisional nonequilibrium. Speedup factors of 2.29, 2.34, and 2.59 were obtained for the $\mathbf{M 1 2}, \mathbf{M 1 2} \mathbf{E}$, and $\mathbf{M} 12_{\mathbf{R}}$ cases, respectively, while using $98 \%$, $76 \%$, and $59 \%$ of the memory required by a full DSMC simulation.

In order to further increase the speedup factor, rotational relaxation should be incorporated within the MPC method. While existing for both DSMC and CFD codes, ${ }^{29}$ it is only currently invoked in DSMC regions. By using rotational relaxation in the CFD module, the CFD domain can be enlarged, and therefore, the DSMC domain would decrease which would lead to a direct reduction in the number of particles required for the MPC method. In addition, in order to apply the MPC method to higher enthalpy flows, multiple species and finite-rate chemistry should be incorporated within the MPC method. Both DSMC and CFD already have the ability to simulate both multiple species and finite-rate chemistry, but the control of the statistical scatter and information transfer associated with the physical process requires additional research. 


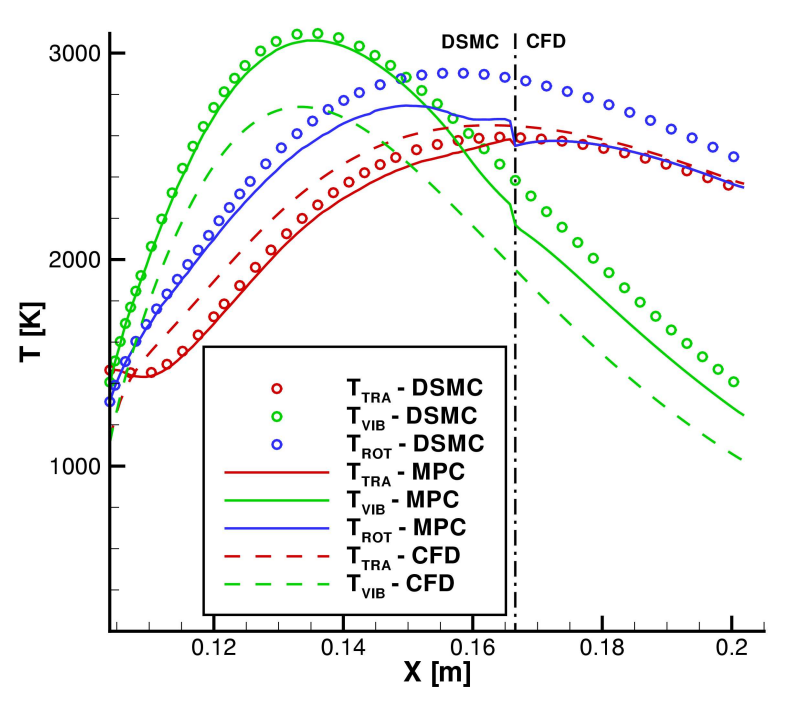

(a) M12 case.

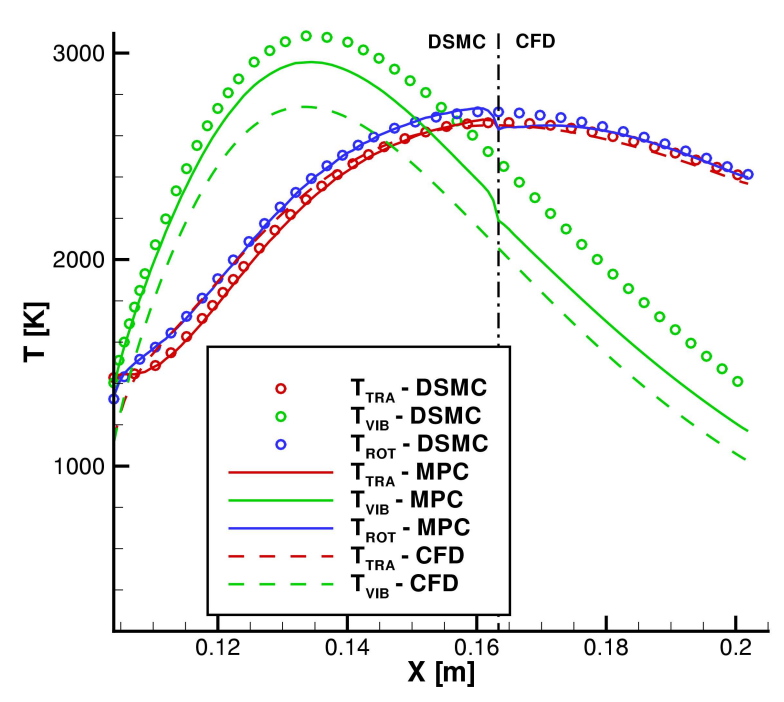

(b) $\mathrm{M} 12_{E}$ case.

Figure 8. Comparison of temperature profiles along the $135^{\circ}$ extraction line.

In addition, differences in the vibrational temperature flow field could cause added discrepancy in finite-rate chemistry due to the coupled vibration-dissociation process.

\section{Acknowledgments}

The first two authors gratefully acknowledge funding from NASA Grant NCC3-989. The third author acknowledges support from the Air Force Office of Scientific Research (AFOSR) under Grant No. FA955004-1-0341. The views and conclusions contained herein are those of the authors and should not be interpreted as necessarily representing the official policies or endorsements, either expressed or implied, of the AFOSR or the U.S. Government.

\section{References}

${ }^{1}$ Bird, G. A., Molecular Gas Dynamics and the Direct Simulation of Gas Flows, Clarendon Press, 1994.

${ }^{2}$ Glass, C. E. and Gnoffo, P. A., "Comparison of a 3-D CFD-DSMC Soultion Methodology With a Wind Tunnel Experiment," Tech. Rep. TM-2002-211777, NASA, August 2002.

${ }^{3}$ Glass, C. E. and Gnoffo, P. A., "A 3-D Coupled CFD-DSMC Solution Method With Application to the Mars Sample Return Orbiter," Tech. Rep. TM-2000-210322, NASA, July 2000.

${ }^{4}$ Wilmoth, R. G., Mitcheltree, R. A., Moss, J. N., and K., D. V., "Zonally Decoupled Direct Simulation MOnte Carlo Solutions of Hypersonic Blunt-Body Flows," Journal of Spacecraft and Rockets, Vol. 31, No. 6, 1994, pp. 971-979.

${ }^{5}$ Hash, D. B. and Hassan, H. A., "Assessment of Schemes for Coupling Monte Carlo and Navier-Stokes Soultion Methods," Journal of Thermophysics and Heat Transfer, Vol. 10, No. 2, 1996, pp. 242-249.

${ }^{6}$ Hash, D. B. and Hassan, H. A., "A Decoupled DSMC/Navier-Stokes Analysis of a Transitional Flow Experiment," Collection of Technical Papers - 34th AIAA Aerospace Sciences Meeting and Exhibit, Vol. 1, Reno, NV, 1996.

${ }^{7}$ Roveda, R., B., G. D., and Varghese, P. L., "Hybrid Euler/Direct Simulation Monte Carlo of Unsteady Slit Flow," Journal of Spacecraft and Rockets, Vol. 37, No. 6, 2000, pp. 753-760.

${ }^{8}$ Roveda, R., B., G. D., and Varghese, P. L., "Hybrid Euler/Particle Approach for Continuum/Rarefied Flows," Journal of Spacecraft and Rockets, Vol. 35, No. 3, 1998, pp. 258-265.

${ }^{9}$ Wijesinghe, H. S. and G., H. N., "A discussion of Hybrid Atomistic-Continuum Methods for Multiscale Hydrodynamics," International Journal for Multiscale Computational Engineering, Vol. 2, 2004.

${ }^{10}$ Schwartzentruber, T. E. and Boyd, I. D., "A hybrid particle-continuum method applied to shock waves," Journal of Computational Physics, Vol. 215, No. 2, July 2006, pp. 402-416. 


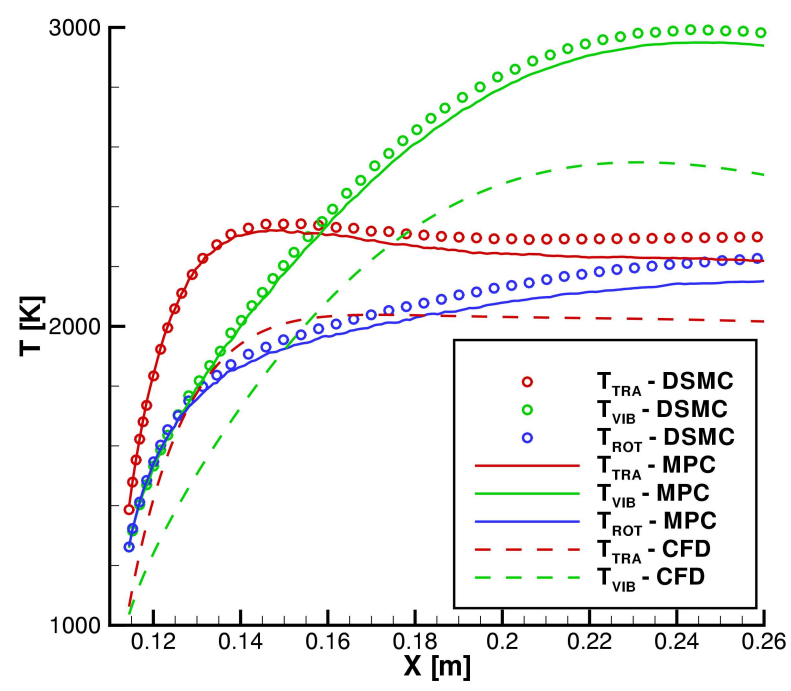

(a) M12 case.

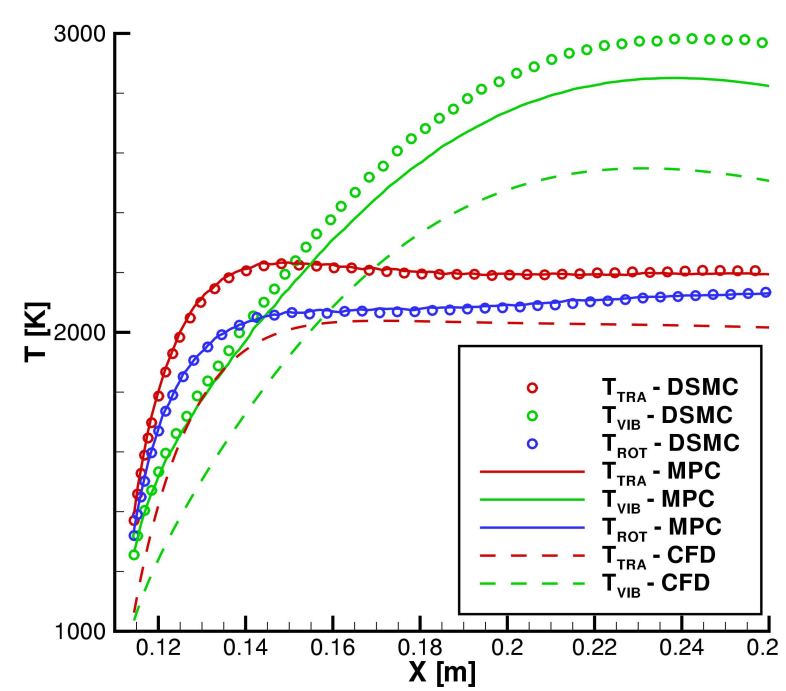

(b) $\mathrm{M} 12_{E}$ case.

Figure 9. Comparison of temperature profiles along the $165^{\circ}$ extraction line.

\footnotetext{
${ }^{11}$ Schwartzentruber, T. E., Scalabrin, L. C., and Boyd, I. D., "Hybrid particle-continuum simulations of nonequilibrium hypersonic blunt-body flowfields," Journal of Thermophysics and Heat Transfer, Vol. 22, No. 1, 2008, pp. $29-37$.

${ }^{12}$ Schwartzentruber, T. E., Scalabrin, L. C., and Boyd, I. D., "A modular particle-continuum numerical method for hypersonic non-equilibrium gas flows," Journal of Computational Physics, Vol. 225, No. 1, July 2007, pp. 1159-1174.

${ }^{13}$ Schwartzentruber, T. E., Scalabrin, L. C., and Boyd, I. D., "Modular implementation of a hybrid DSMC-NS algorithm for hypersonic non-equilibrium flows," Collection of Technical Papers - 45th AIAA Aerospace Sciences Meeting, Vol. 11, Reno, NV, United States, 2007, pp. 7449-7461.

${ }^{14}$ Dietrich, S. and Boyd, I. D., "Scalar and Parallel Optimized Implementation of the Direct Simulation Monte Carlo Method," Journal of Computational Physics, Vol. 126, No. 2, July 1996, pp. 328-342.

${ }^{15}$ Boyd, I. D., "Analysis of rotational nonequilibrium in standing shock waves of nitrogen," AIAA Journal, Vol. 28, No. 11, 1990, pp. 1997-1999.

${ }^{16}$ Scalabrin, L. C. and Boyd, I. D., "Development of an Unstructures Navier-Stokes Solver for Hypersonic Nonequilibrium Aerothermodynamics," 38th AIAA Thermophysics Conference, Toronto, Canada, June 6-9 2005.

${ }^{17}$ Millikan, R. C. and White, D. R., "Systematics of Vibrational Relaxation," The Journal of Chemical Physics, Vol. 39, No. 12, December 1963, pp. 3209-3213.

${ }^{18}$ Park, C., Nonequilibrium Hypersonic Aerothermodynamics, John Wiley \& Sons, 1990.

${ }^{19}$ Boyd, I. D., "Analysis of vibrational-translational energy transfer using the direct simulation Monte Carlo method," Phys. Fluids A, Vol. 3, No. 7, July 1991, pp. 1785-1791.

${ }^{20}$ Farbar, E. D., "Testing of Vibrational Relaxation Model in MONACO," Department of Aerospace Engineering, University of Michigan.

${ }^{21}$ Lofthouse, A. J., Scalabrin, L. C., and Boyd, I. D., "Hypersonic aerothermodynamics analysis across nonequilibrium regimes using continuum and particle methods," Collection of Technical Papers - 39th AIAA Thermophysics Conference, Vol. 1, Miami, FL, United States, 2007, pp. 103-126.

${ }^{22}$ Vijayakumar, P., Sun, Q., and Boyd, I. D., "Vibrational-translational energy exchange models for the direct simulation Monte Carlo method," Phys. Fluids, Vol. 11, No. 8, Aug. 1999, pp. 2117-2126.

${ }^{23}$ Abe, T., "Direct Simulation Monte Carlo method for internal-translational energy exchange in nonequilibrium flow," Rarefied Gas Dynamics: Theory and Simulations, 1994.

${ }^{24}$ Larsen, P. S. and Borgnakke, C., "Statistical Collision Model for Simulating Polyatomic Gas with Restricted Energy Exchange," Soviet Powder Metallurgy and Metal Ceramics (English translation of Poroshkovaya Metallurgiya), 1974, pp. 9-.

${ }^{25}$ Boyd, I. D., Chen, G., and Candler, G. V., "Predicting failure of the continuum fluid equations in transitional hypersonic flows," Phys. Fluids, Vol. 7, No. 1, Jan. 1995, pp. 210-219.

${ }^{26}$ Wang, W.-L. and Boyd, I. D., "Predicting continuum breakdown in hypersonic viscous flows," Phys. Fluids, Vol. 15, No. 1, Jan. 2003, pp. 91-100.

${ }^{27}$ Sun, Q. and Boyd, I. D., "Evaluation of Macroscopic Properties in the Direct Simulation Method," Journal of Thermophysics and Heat Transfer, Vol. 19, No. 3, 2005, pp. 329-335.
} 


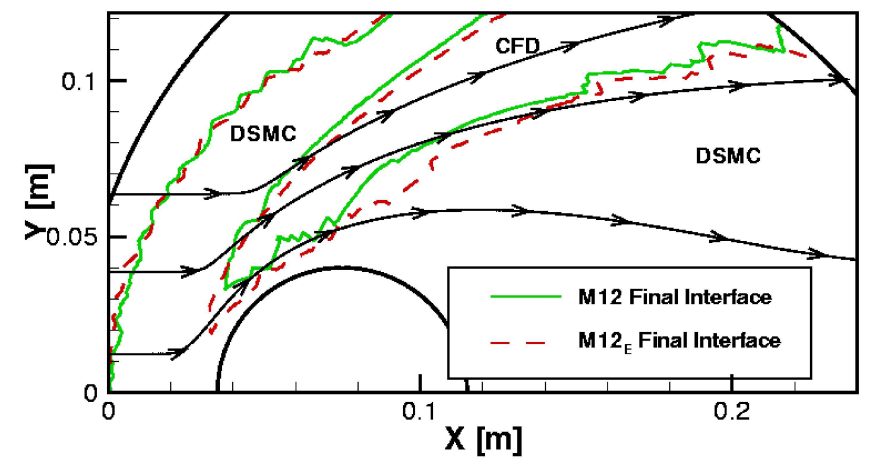

Figure 10. Interface locations with streamlines that pass through the areas that exhibit largest discrepancy in vibrational temperature between the MPC method and DSMC.

${ }^{28}$ Lofthouse, A. J., Scalabrin, L. C., and Boyd, I. D., "Hypersonic Aerothermodynamics Analysis Across Nonequilibrium Regimes Using Continuum and Particle Methods," Collection of Technical Papers - 39th AIAA Thermophysics Conference, Vol. 1, Miami, FL, United States, 2007.

${ }^{29}$ Holman, T. D. and Boyd, I. D., "Numerical Investigation of the Effects of Continuum Breakdown on Hypersonic Vehicle Surface Properties," AIAA Paper 2008-3928, Seattle, WA, United States, 2008. 


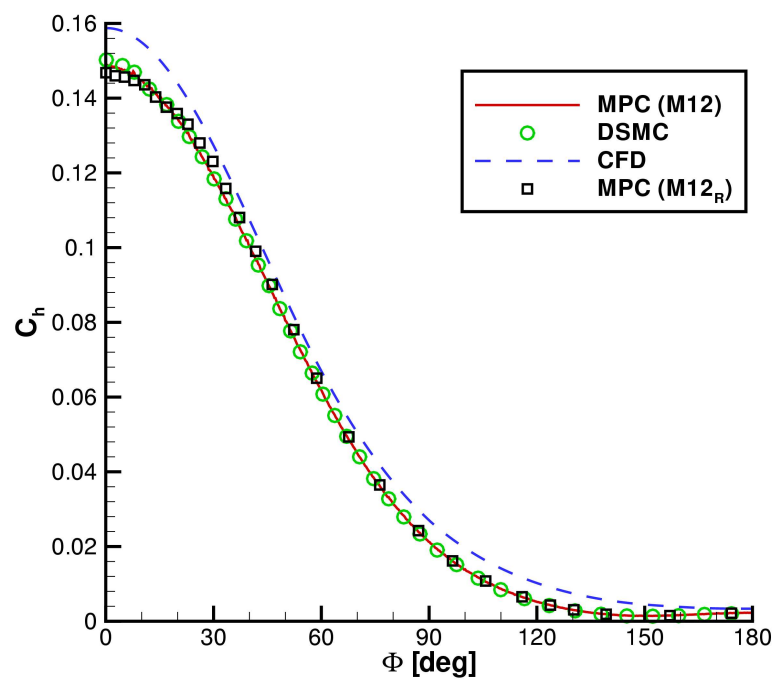

(a) M12 and M12 $R$ cases.

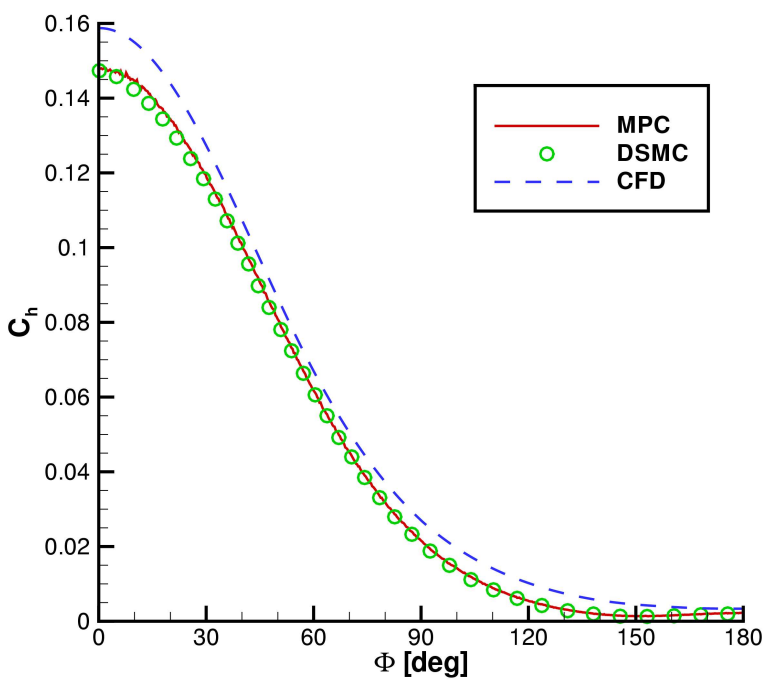

(b) $\mathrm{M} 12_{E}$ case.

Figure 11. Comparison of coefficient of heating between CFD, DSMC and MPC. 